\title{
The new discharge hopper and EDEM simulation analysis for particles flat accumulation after free falling
}

\author{
Jianming Yuan, Hailing Yao, Zhihui $\mathrm{Hu}^{\mathrm{a}}$ and Jie Zhou \\ School of Logistics Engineering, Wuhan University of Technology, wuhan, 430063, hubei, China
}

\begin{abstract}
Put forward a new type discharge hopper, for material free falling uneven stacking problems because of now commonly used the cone square opening shape discharge hopper, by setting the dosing plate and the guide plate, change the material particles flow velocity and flow direction on discharge outlet. Through simulation analysis the material free falling piling process of pulverized coal, can get the rule of change new discharge hopper structure parameters will influence the material falling efficiency and the flat accumulation, the simulation results show that using the new discharge hopper to material free falling accumulation, can guarantee the material falling efficiency, realize the ideal flat accumulation at the same time.
\end{abstract}

Keywords: discharge hopper; discrete element method; EDEM simulation.

\section{Introduction}

Nowadays, we often use cone square opening shape discharge hopper leading granular materials transport, transfer heap and loading process. In the process of discharge, the granular materials outflow under the action of gravity, in flow process, the fiction down non-uniform to adapt the change of cross section shrinkage. Due to the closer the discharge outlet, the bigger hopper cross section shrinkage, it will increase the force of friction between particles, at the discharging port as to achieve the maximum of the internal and external force of friction. At this time, above the discharge outlet to form a tubular discharge channel, the flow velocity of granular materials is larger in the channel, when far from the discharging channel the flow velocity will become smaller [1]. Because of the flow characteristics of granular materials, it will form pointed horn stock pile after material falling go through cone square mouth shape discharge hopper. This pointed horn windrow will reduce space utilization rate, and in the process of non-uniform velocity transportation, such as trains and ships, stock pile will appear not stable state due to the effect of external causes, such as vibration, inertia force, centrifugal force, and so on. It will lead to carrying tools unbalance loading, affects normal transport operations. So the granular materials falling accumulation pass straight square opening, also need to take artificial and mechanical measures flat stock pile [2].

For now commonly exist problem of the cone straight square opening discharge hopper[3] uneven stacking, this paper puts forward a new type discharge hopper, the discharge hopper by setting the dosing plate and the guide plate, change the material particles flow velocity and flow direction on discharge outlet, realize flat accumulation after material falling naturally. And adopted at present are

\footnotetext{
${ }^{\mathrm{a}}$ Corresponding author : 565337283@qq.com
} 
widely used in the particle motion analysis of EDEM simulation software[4,5] simulation analysis the rule of flow velocity and flow direction change effect the material falling efficiency and flat accumulation.

\section{The new discharge hopper structure}

Through researching and analyzing the materials flow characteristics of the discharge hopper material falling process, existing cone square opening shape discharge hopper is proposed an improvement program as shown in Figure 1.

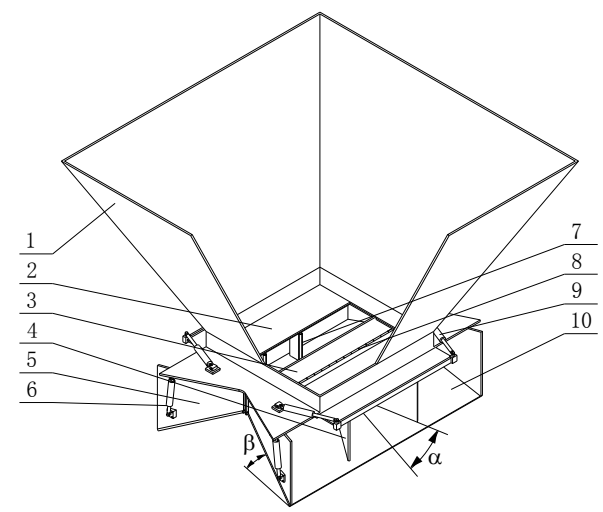

1- discharge hopper cone machine body 2- discharge outlet opening control plate 3- Discharge outlet partition plate 4- dosing plate 5- side fences 6- guide trough rotating pusher 7- opening control separator putter 8- dosing plate shaft 9- guide plate shaft 10- guide plate

Figure 1. The new discharge hopper structure

Discharge hopper structure is symmetrical, in which the opening and closing of discharge outlet opening control plate is controlled by pushing, dosing plate driven by motor and rotating about its axis of rotation, the guide plate also rotating about its axis of rotation. Discharge outlet partition plate is bolted to the discharge hopper cone machine body. The shaft bearing of guide plate is secured to the blanking opening partition plate. Side of the baffle is fixedly connected with the guide plate, together constitute the guiding groove, while the push rod is rotated through the guide chute to connect with discharge hopper cone hopper body, and the entire guide troughs are controlled and rotated by pushing. The sub-plate and the side angle is supposed to $\alpha$, the guide plate and the horizontal angle is supposed to $\beta$.

\section{Establish EDEM simulation model}

\subsection{Material and contact geometry parameters}

This paper choose more representative of granular materials-pulverized coal as research subjects. In EDEM simulation software, the parameters of the material particles is set to pulverized coal parameters, and the materials of carriage and discharge hopper are set to steel. The simulation parameter settings as shown in table 1 . 
AEST2016

Table 1. Simulation parameters

\begin{tabular}{ll}
\hline Parameters & Value \\
\hline Poisson's ratio of pulverized coal & 0.3 \\
Steel Poisson's ratio & 0.3 \\
Pulverized shear modulus $(\mathrm{Pa})$ & $1 \mathrm{e}+07$ \\
Steel shear modulus $(\mathrm{Pa})$ & $1 \mathrm{e}+08$ \\
Pulverized coal density $\left(\mathrm{kg} / \mathrm{m}^{3}\right)$ & 1300 \\
Steel density $\left(\mathrm{kg} / \mathrm{m}^{3}\right)$ & 7850 \\
Coal and coal recovery restitution & 0.5 \\
Steel and coal recovery restitution & 0.5 \\
Coal and coal coefficient of static friction & 0.5 \\
Steel and coal coefficient of static friction & 0.5 \\
Coal and coal coefficient rolling friction & 0.2 \\
Steel and Coal coefficient rolling friction & 0.05 \\
\hline
\end{tabular}

\subsection{Particles model and contact model}

Due to the complex shape of the coal particles, different and very irregular the particle sizes, for convenience, to meet the premise of the particles performance requirements, the particle shape is set to ellipsoidal in simulation process. As shown in Figure 2, particles are combined by three spherical model, directly synthesized in EDEM software, and the entire simulation as both particles are particles prototyping.

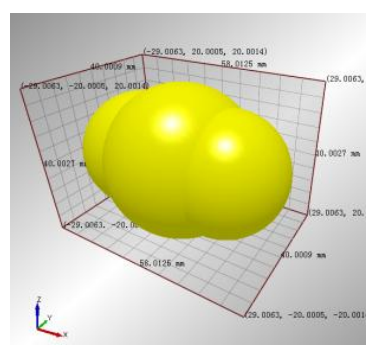

Figure 2. Particle model

Selection of the contact model is directly related to the accuracy of the results. EDEM software itself offers nine models to choose, and each contact model has its scope. Taking into account the moisture content of coal is very low, its nearly ideal granules, so choose the Hertz-Mindlin contact model.

\section{EDEM simulation analysis}

\subsection{Determination of the simulation variables}

In this paper, the simulation by changing the discharge hopper structure a variety of different forms are obtain multiple sets of simulation results corresponding. In theory, the mass flow rate and flatness of material falling can be controlled by respectively control the size of the discharging outlet opening size or Angle of $\alpha$ and $\beta$, but because of the discharge outlet be divided into two parts and add the guide plate will greatly reduce the material flow, if the discharge outlet size is too small may appear material plugging phenomenon in discharge outlet, so not control the discharge outlet opening size to control the mass flow rate and flatness of material falling, discharge outlet opening control plate just as open and close.

In order to make the material can be falling, guide plate Angle should be the minimum to ensure the material can be sliding down along the guide plate: 


\section{AEST2016}

$$
\beta>\arctan \mu
$$

In formula: $\beta$ is the angle of guide plate and the horizontal plane; $\mu$ is the static friction coefficient of coal and steel. After calculation, $\beta>26.6^{\circ}$, taking the minimum value of $\beta$ is $30^{\circ}$.

In this simulation, the car should be moving at a constant speed along the length direction in order to assure the uniformity of material falling along the length direction, carriage movement speed calculated by formula:

$$
t_{1}=\frac{m_{\mathrm{z}}}{Q_{m}} \text { and } v_{c}=\frac{s}{t_{1}}
$$

In formula: $\mathrm{t}_{1}$ is the time of material falling; $\mathrm{m}_{\mathrm{z}}$ is the overall quality of material; $\mathrm{Q}_{\mathrm{m}}$ is the mass flow rate of discharge outlet; $v_{c}$ is carriage movement speed, $s$ is the displacement of carriage.

\subsection{Simulation analysis of falling efficiency}

Respectively simulation analysis for dosing plate angle from $0^{\circ}$ to $40^{\circ}$, every $10^{\circ}$ as a group; guide plate angle from $55^{\circ}, 30^{\circ}$ to every $5^{\circ}$ as a group.

Material fall out from the hopper and the process of accumulate shown in Figure 3, including (1) graph is material falling process of square opening shape discharge hopper, (2) graph is material falling process of new type discharge hopper. It can be seen from the diagram after add the tilted guide plate and dosing plate, the material flatness has made a lot of improvement in width direction.

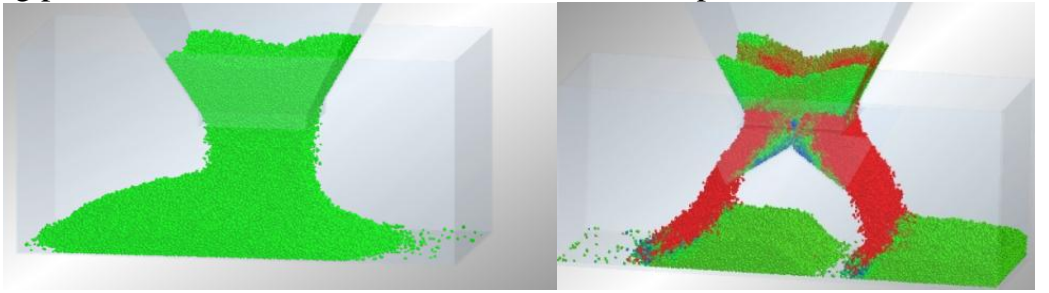

(1)Material falling process square opening shape discharge hopper (2) Material falling process of new type discharge hopper

Figure 3. Falling process contrast

Statistics the mass flow rate of material falling for the various state discharge hopper respectively, analysis of new type discharge hopper falling efficiency features. Take the discharge hopper average mass flow rate when material falling stable as the criteria. The mass flow rate of dosing plate angle of $\alpha$ changes from $0^{\circ}$ to $40^{\circ}$ as shown in Figure 4(a), it can be seen from the diagram mass flow rate showed a trend of decrease with the increase of $\alpha$ angle, but it tend to stabilized between $10^{\circ}$ to $30^{\circ}$. Figure 4(b) is the curve of the mass flow rate with the guide plate angle $\beta$ change when dosing plate angle of $\alpha$ is $20^{\circ}$. It can be seen that with the increase of angle of $\beta$ material falling mass flow rate are increased.
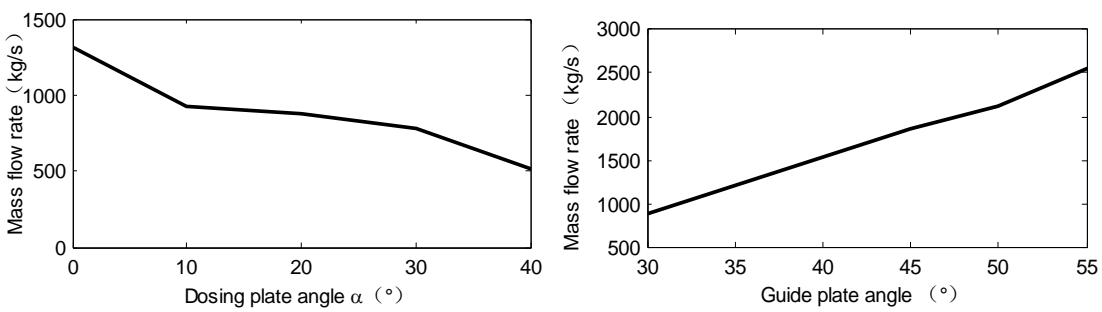

Figure 4. (a)The mass flow rate change with dosing plate Angle curve (b)The mass flow rate change with guide plate angle curve 


\subsection{Simulation analysis of flat accumulation}

Adopt the following methods of quantitative expression of the flatness.

As shown in Figure 5, carriage is divided into finite grid at length and width direction.

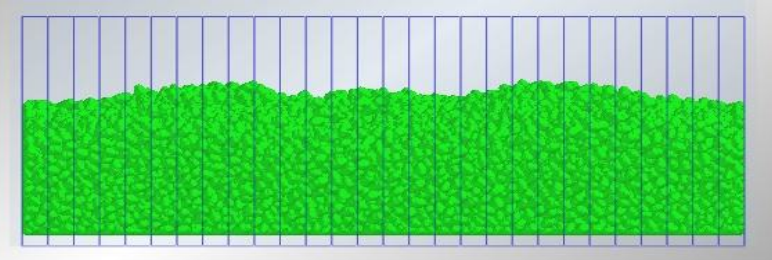

Figure 5. Grid division diagram

In the EDEM, we can get the quality of the material in each grid, and carry on statistics processing. Statistical processing method is as follows:

$$
\begin{gathered}
\sigma_{m}=\sqrt{\frac{1}{N} \sum_{i=1}^{N}\left(m_{i}-E_{m}\right)^{2}} \quad E_{h}=\frac{E_{m}}{\rho A} \\
h=\frac{\sigma_{m}}{\rho A} \quad P=\frac{h}{E_{h}}=\frac{\sigma_{m}}{E_{m}}
\end{gathered}
$$

In formula, $\mathrm{N}$ is number of grid; $\mathrm{m}_{\mathrm{i}}$ is material quality of ith grid. $\mathrm{E}_{\mathrm{m}}$ is average quality of material in all grid; $\sigma_{\mathrm{m}}$ is standard deviation of material quality all of $\mathrm{N}$ grid; $\mathrm{E}_{\mathrm{h}}$ is average height of accumulation; $\rho$ is coal bulk density; $\mathrm{A}$ is area at the bottom of the grid; $\mathrm{P}$ is flatness, the lower its value, the better of flatness.

As shown in Figure 6(a) is flatness change with dosing plate angle curve. The solid line in figure is the length direction accumulation flatness $\mathrm{P}$ with $\alpha$ change curve, it can be seen as dosing plate angle $\alpha$ change its basic do not change. Dotted line is the width direction accumulation flatness $\mathrm{P}$ with $\alpha$ change curve, it can be seen accumulation flatness got a lot of improvement after add dosing plate, and the material accumulation flatness is the best when the dosing plate angle is $10^{\circ} \sim 30^{\circ}$, but it will be gradually decline when the dosing plate angle more than $30^{\circ}$.
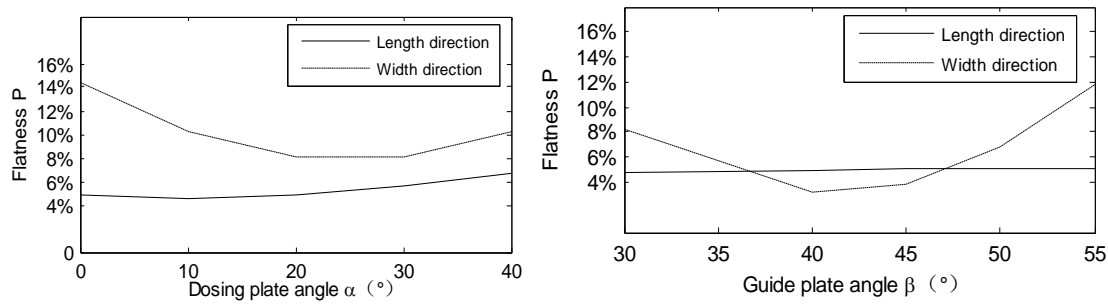

Figure 6. (a)Flatness change with dosing plate Angle curve (b)The flatness with guide plate angle change curve

From the above analysis, selection dosing plate angle is $20^{\circ}$, and Figure $6(\mathrm{~b})$ shows the accumulation flatness with the guide plate angle change curve when the dosing plate is $20^{\circ}$. The solid line in figure is the length direction pile flatness $\mathrm{P}$ with $\beta$ change curve, so it can be seen the presence or absence of guide plate has a small impact on accumulation flatness of the material in the length direction. Dotted line is the width direction accumulation flatness $\mathrm{P}$ with $\beta$ change curve, so it can be seen, with the increase of guide plate angle, accumulation flatness $\mathrm{P}$ in the width direction represents the trend of first decreasing and then increasing, and the material accumulation flatness is the best when the guide plate angle is $40^{\circ} \sim 45^{\circ}$.

Through the above comprehensive analysis, it can be seen the accumulation flatness is best when the dosing plate angle is $20^{\circ}$ and the guide plate angle is $40^{\circ}$ to $45^{\circ}$. 


\section{Conclusion}

1) By increasing dosing plate and guide plate, and setting them appropriate direction, changing material falling direction and flow rate, the material accumulation state can be effectively changed, and flatness of falling accumulation can get better.

2) Through the analysis of the flatness of pulverized coal accumulation effect in the length direction of the carriage in different conditions of dosing plate and guide plate angle, it can be obtain that dosing plate and guide plate angle have no effect on the flatness in the length direction and the flatness depends on the speed stability of the carriage.

3) Through contrasting mass flow rate and accumulation effect during pulverized coal accumulation in different conditions of dosing plate angle, it can be seen that the accumulation effect get the best when dosing plate angle is from $20^{\circ}$ to $30^{\circ}$.

4) Through contrasting mass flow rate and accumulation effect during pulverized coal accumulation in different conditions of guide plate angle when dosing plate angle is $20^{\circ}$, it can be seen that the accumulation effect and falling efficiency get best when guide plate angle is from $40^{\circ}$ to $45^{\circ}$.

5) Appropriate increase of dosing plate angle to increase mass flow rate if there is higher request for the working efficiency but it is not good to the flatness of accumulation.

\section{Acknowledgments}

Supported by National Science and Technology Support Program Project (Grant No. 2015BAF06B05)

\section{References}

1. WANG Xuewen, QIN Yi, TIAN Yan-kang, YANG Xin-yu, Analysis on flow features of bulk coal during coal unloading period based on EDEM [J].Coal Science and Technology, 2015,43(5):130-134

2. Yuan Jianming, Wang Yonglong, Li Yongzhi, Yang Zhong, Development and Application of Spiral Flattening Machine for Particulate Materials in the Bulk Terminal [J]. Port Operation 2014,218(5):11-14

3. LU Zhenzhou, Research on Unloading Efficiency and its Influencing Factor of Hopper Vehicle [D]. Central South University, 2014

4. H.G.Matuttis, S.Luding,H.J.Herrmann. Discrete element simulations of dense packing and heaps made of spherical and non-spherical particles [J]. powder Technology,2000,109(1-3):278-292

5. Y.C.Zhou, B.D.Wright, R.Y.Yang, B.H.Xu, A.B.Yu. Rolling friction in the dynamic simulation of sandpile formation [J]. Physica A, 1999, 269(2-4): 536-553 